\title{
Image slicer module for Wide Field Optical Spectrograph (WFOS)
}

Shinobu Ozaki, Satoshi Miyazaki, Toshihiro Tsuzuki, Jason R. Fucik

Shinobu Ozaki, Satoshi Miyazaki, Toshihiro Tsuzuki, Jason R. Fucik, "Image slicer module for Wide Field Optical Spectrograph (WFOS)," Proc. SPIE 10702, Ground-based and Airborne Instrumentation for Astronomy VII, 107028M (11 July 2018); doi: 10.1117/12.2309324

Event: SPIE Astronomical Telescopes + Instrumentation, 2018, Austin, Texas, United States 


\title{
Image slicer module for Wide Field Optical Spectrograph (WFOS)
}

\author{
Shinobu Ozaki ${ }^{\mathrm{a}}$, Satoshi Miyazaki ${ }^{\mathrm{a}}$, Toshihiro Tsuzuki ${ }^{\mathrm{a}}$, Jason R. Fucik ${ }^{\mathrm{b}}$ \\ ${ }^{a}$ National Astronomical Observatory of Japan, Osawa, Mitaka, Tokyo, 181-8588, Japan; \\ ${ }^{\mathrm{b}}$ Caltech Optical Observatories, California Institute of Technology, Pasadena, CA 91125, USA;
}

\begin{abstract}
Wide-Field Optical Spectrograph (WFOS) is an optical multi-object spectrograph and one of the first-light instruments of Thirty Meter Telescope (TMT). The WFOS development team has studied three new instrument concepts. One is a fiber-based spectrograph, and other one is a spectrograph using image slicers (Slicer-WFOS). The last one is the simple multi-slit spectrograph. Japanese WFOS team has conducted conceptual studies on Slicer-WFOS in collaboration with California Institute of Technology. Slicer-WFOS has only one VPH grating for each red and blue arm. The gratings offer $\mathrm{R} \sim 1,500$ for a simple $0 . " 75$-width slit. The image slicer divides an object image into three slices and the higher spectral resolution of $\mathrm{R} \sim 4,500$ can be achieved using the same grating. In this proceeding paper, we report our design studies on the slicer module.
\end{abstract}

Keywords: Image slicer, Wide Field Optical Spectrograph (WFOS), Thirty Meter Telescope (TMT)

\section{INTRODUCTION}

Wide-Field Optical Spectrograph (WFOS) ${ }^{[1,2,3]}$ is one of the first-light instruments of Thirty Meter Telescope (TMT), and has been developed in an international collaboration led by University of California Observatories. Its spectral resolving powers (R) are $\sim 5,000$ and $\sim 1,000$ in the high dispersion mode and the low dispersion mode, respectively. WFOS is wanted to cover the wavelength range of $310-1,000 \mathrm{~nm}$ simultaneously even in the high dispersion mode. In the previous baseline concept, WFOS had cross-dispersion prisms in addition to the main reflective gratings in order to observe some consecutive spectral orders simultaneously. However, we identified possible concerns for this concept such as a large ( $\mathrm{D} \sim 440 \mathrm{~mm}$ ) complex refractive camera and a large vignetting at the field edge due to a large distance between the main disperser and the first surface of the camera.

To avoid these concerns, we started to study alternate concepts ${ }^{[4]}$. Japanese WFOS team is studying one of the concepts using image slicers (Slicer-WFOS) in collaboration with California Institute of Technology. Slicer-WFOS has only one transmission VPH grating for each red and blue arm (Figure 1). The gratings offer R 1,500 for simple 0."75-width slits. In the high spectral dispersion mode, small image slicer modules are put on the telescope focal surface instead of a slit mask. The image slicer divides object image into three slices, and the higher spectral resolution of $\mathrm{R} \sim 4,500$ can be achieved using the same grating. The transmission VPH grating allows us to locate the cameras closer to the input pupils. In addition, the input pupil shapes keep circular with a $300-\mathrm{mm}$ diameter because the gratings are used in the Littrow configuration. These features make the camera optical design much easier and the vignetting lower.

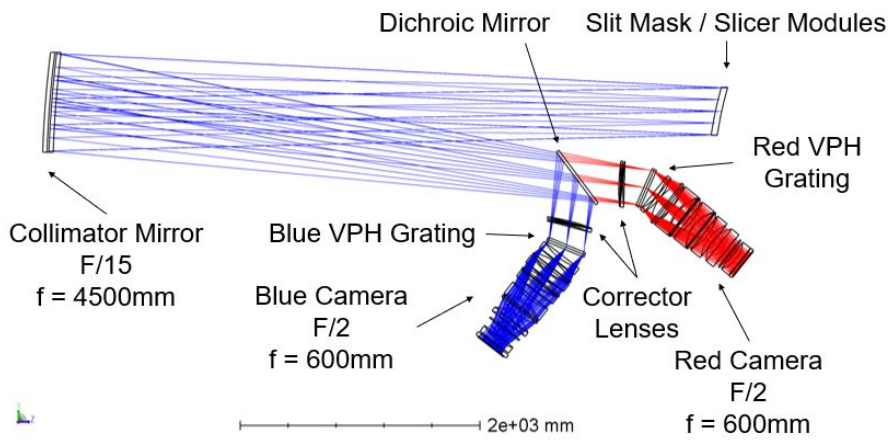

Figure 1. Optical layout of Slicer-WFOS.

Ground-based and Airborne Instrumentation for Astronomy VII, edited by Christopher J. Evans, Luc Simard, Hideki Takami, Proc. of SPIE Vol. 10702, 107028M - @ 2018 SPIE · CCC code: 0277-786X/18/\$18 · doi: 10.1117/12.2309324 
Japanese WFOS team has conducted a design study of the slicer module. The results are reported in this paper. We developed three optical designs for the slicer module and those are introduced in the section 2. In the Slicer-WFOS concept, TMT field curvature imposes us a technical challenge. We explain the challenge and our solution in the section 3.

\section{OPTICAL DESIGN}

Basic requirements for the image slicer are summarized in Table 1. The slice width is $0 . " 25$, one-third of the putative seeing size (0."75). The slice length is 7", which is required for precise sky subtraction using a nodding method. SlicerWFOS accepts the total slit length of 8.'3, and hence the maximum multiplexity is 23 considering some margin for optomechanics.

The requirement for the output beam direction change with respect to the original direction is introduced. TMT does not have adequate baffles, and therefore WFOS will have an internal baffle in order to cut stray light. The baffle will be located around the pupil image. If the output beam direction significantly changes from the input beam, the pupil image significantly shifts and then the baffle causes larger vignetting and larger stray light comes in the baffle aperture. The requirement of $<0.115$ degree allow 3\% pupil shift. This value is now temporal and must be revised in future.

Table 1. Basic requirements for the image slicer.

\begin{tabular}{|l|l|}
\hline Slice width & $0 . " 25(0.55 \mathrm{~mm})$ \\
\hline Slice length & 7 " $(14 \mathrm{~mm})$ \\
\hline Slice number & 3 \\
\hline Output beam direction change & $<0.115$ degree \\
\hline
\end{tabular}

\subsection{Two-mirror design}

To cooperate with simple slit masks for the low-dispersion mode, the slicer module is required to provide the exit pupil and the exit $\mathrm{F}$ ratio same as those of the telescope, and the pseudo slits are also required to be at the telescope focal surface. Since those requirements can be controlled with at least two surfaces, we started with the two-mirror system. Figure 2 shows the optical design. All mirrors have concave spherical surfaces with an identical curvature radius. For the central slice, there is no slice mirror, and incoming light passes through the module. Incoming light to the two side slices
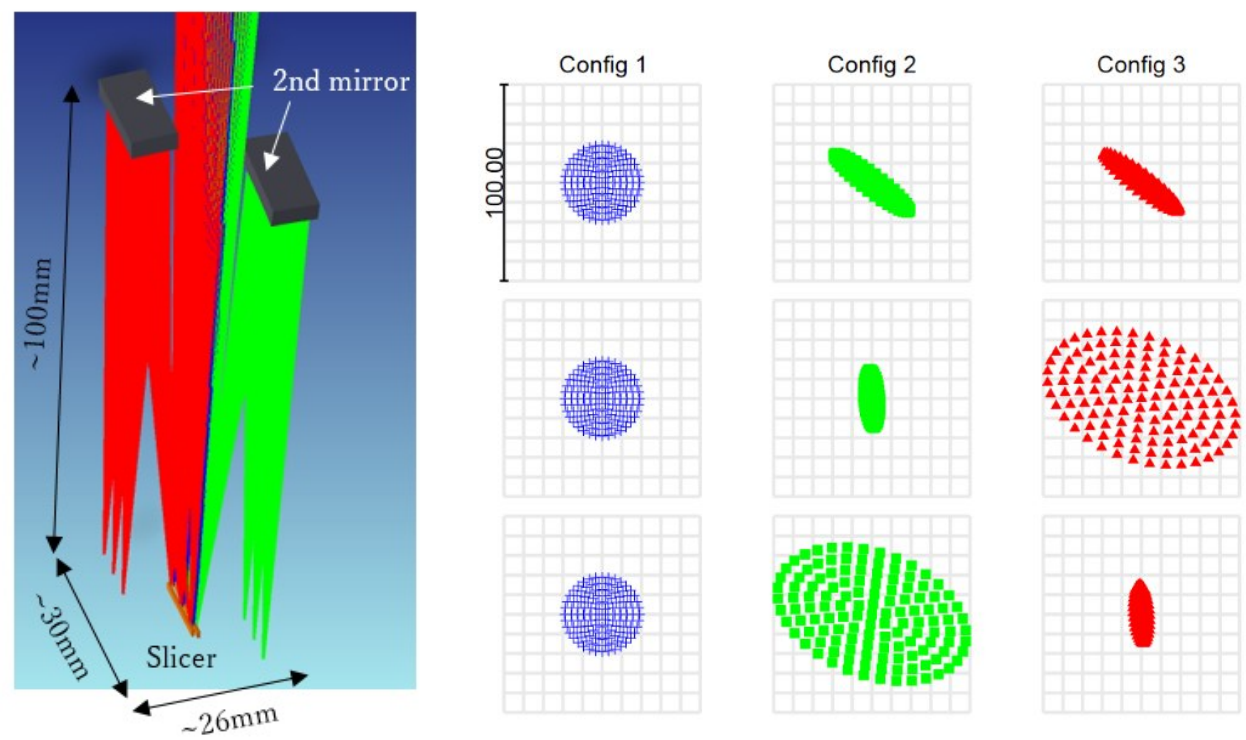

Figure 2. Optical layout (left) and spot diagram of the two-mirror design (right). In both panels, blue shows light rays and spots for the central slice. Green and red show those of the two side slices. In the spot diagram, the box size is $100 \mu \mathrm{m}(\sim 0 . " 046)$. 
are reflected by the slice mirrors, and reimaged next to the center slice at the telescope focal plane by the secondary mirrors. For easy manufacturing, actual slice mirrors have larger size than the reflection area and are masked for unused area (Figure 3).

Although this design shows sufficient image quality, large module-tilt sensitivity was found in the output beam direction. The output beam tilts by about the double of the module tilt. For example, when the module tilts by 0.5 degree, the output beam tilts by about 1 degree with respect to the original direction.

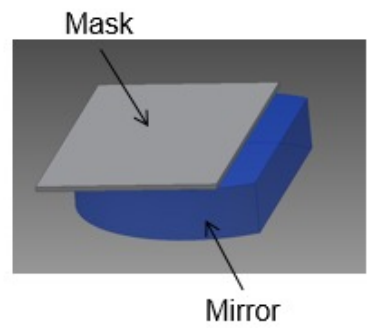

Figure 3. Slice mirror concept. A larger mirror is masked except the slice mirror area.

\subsection{Four-mirror design}

To reduce the module-tilt sensitivity, two more mirrors were added for each channel (Figure 4). Similar to the two-mirror design, all mirrors have concave spherical surfaces with an identical curvature radius. In this design, the last two mirrors cancel the sensitivity by the first two mirrors. Thank to this feature, the module-tilt sensitivity of the output beam direction is dramatically improved. For the module-tilt of 0.5 degree, the output beam tilt only by 0.05 degree with respect to the original direction.

Spot RMS diameter for this four-mirror design is $\sim 50$ um $(\sim 0 . " 023)$ at the telescope focal surface which is not significant comparing with the putative seeing size $(\sim 0 . " 75)$.
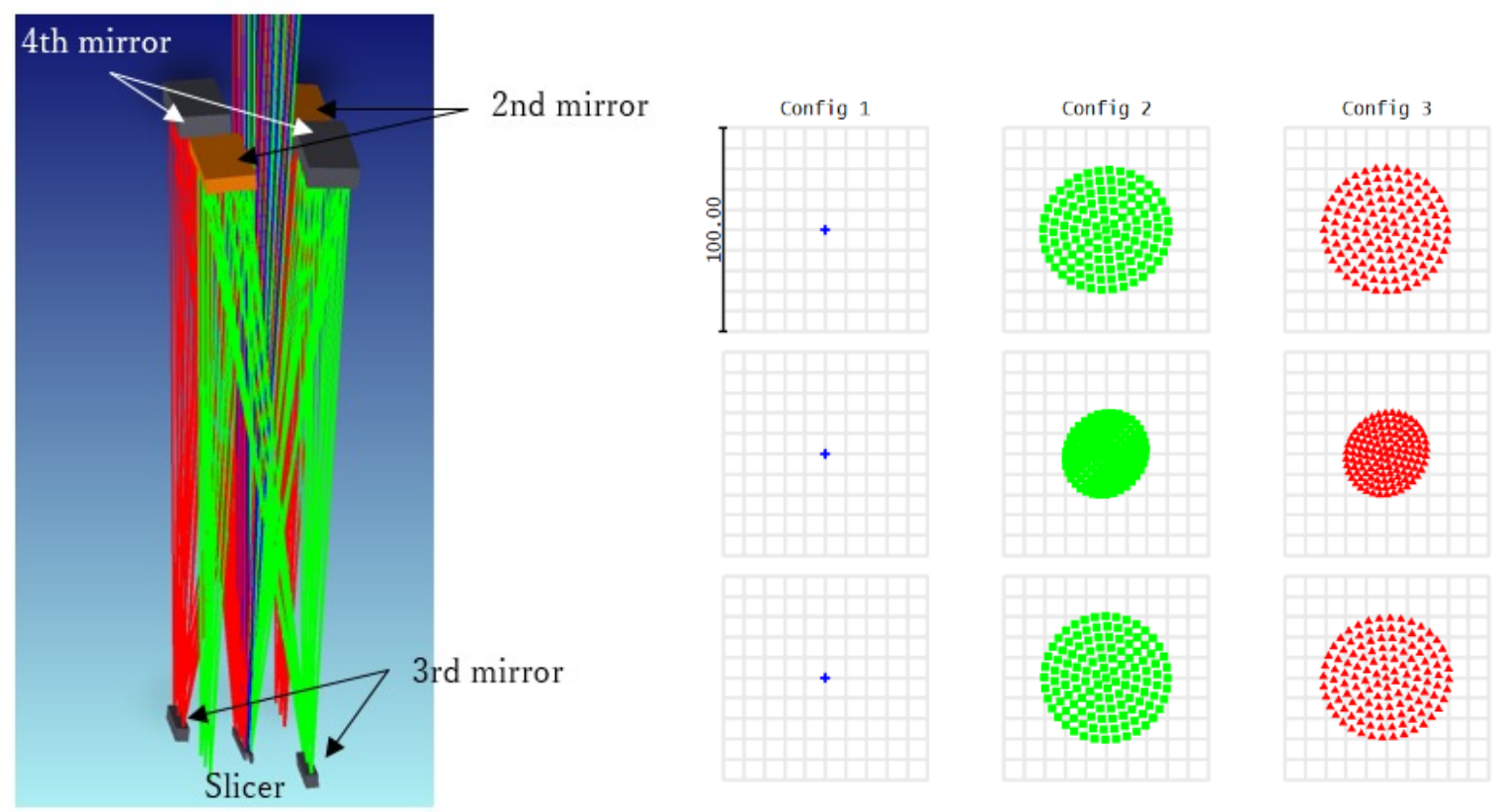

Figure 4. Optical layout (left) and spot diagram of the four-mirror design (right). In both panels, blue shows light rays and spots for the central slice. Green and red show those of the two side slices. In the spot diagram, the box size is $100 \mu \mathrm{m}(\sim 0 . " 046))$. 
Because the number of mirrors increases, uncertainty of the output beam direction is expected to be larger due to the assembly error for each mirror. Figure 5 shows the tolerance analysis result for the output beam direction using a Monte Carlo method. In this analysis, each mirror tilt and dicenter are assumed to be within 0.02 degree and $30 \mu \mathrm{m}$, respectively. It was found that the provability for achieving the requirement of the output beam direction is $\sim 50 \%$. To improve this, higher assembling accuracy is needed, but it is quite challenging.

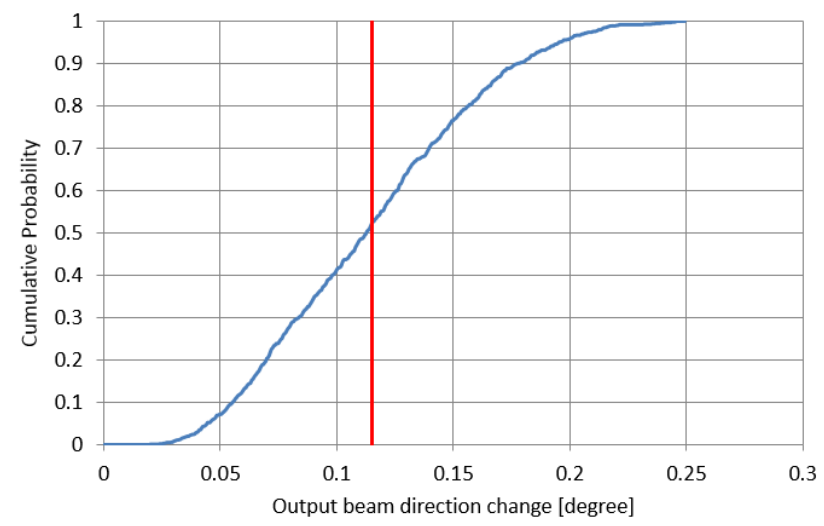

Figure 5. Tolerance analysis result for the four-mirror design. Red line shows the requirement ( 0.115 degree).

\subsection{Flat-mirror system}

To meet the specification of the output beam direction, a completely new design was introduced (Figure 6). In this design, all mirrors are flat. Similar to the other designs, incoming light for the two side slices are reflected by the slice mirrors and reflected again by the secondary mirror. Viewing from the spectrograph, the virtual pseudo-slit images are located behind the secondary mirror. Incoming light for the central slice passes thorough the image slicer and goes through 4 mirrors to match the pseudo-slit location to the other two.

Because flat mirrors are completely insensitive to their dicenters, we can expect better performance in the output beam direction change due to the assembly errors (In the case of concave mirror, the dicenters of each mirror contribute to the output beam direction change as well as their tilts.). For the central channel, the first and the forth mirrors are put on the both surfaces of a wedged glass plate so that assembly error of this wedged plate is canceled out. According to the tolerance analysis with the same parameter ranges as Figure 5, the probability for achieving the requirement of the output beam direction change is almost $100 \%$ (Figure 7).
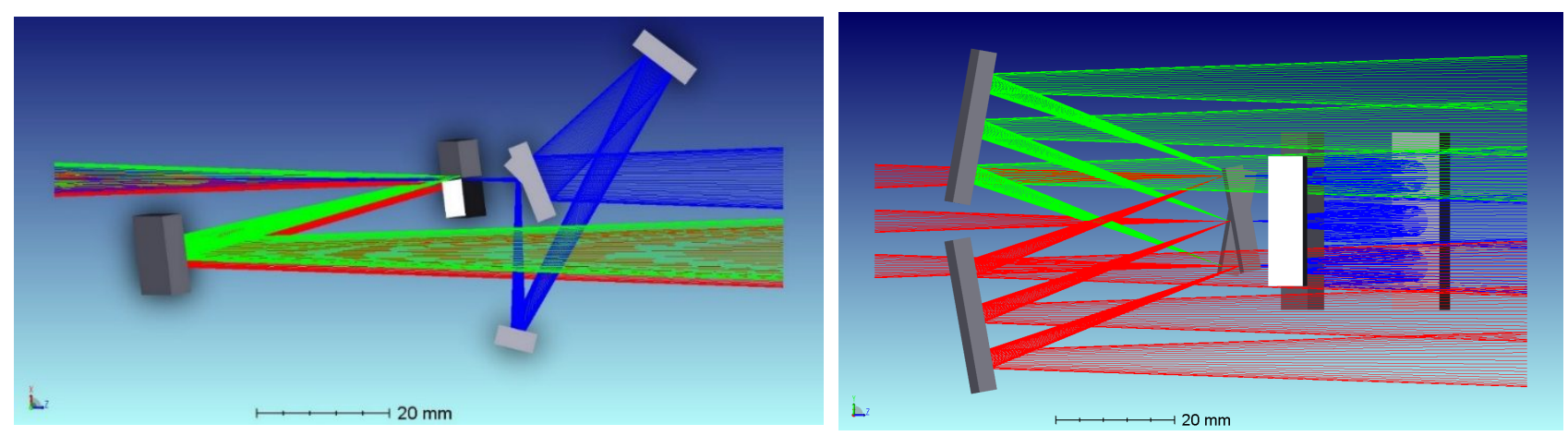

Figure 6. Optical design of the flat-mirror design. Light from the telescope comes from the left side. Blue rays show the light of the central slice. Green and red rays show the light of the two side slices. 


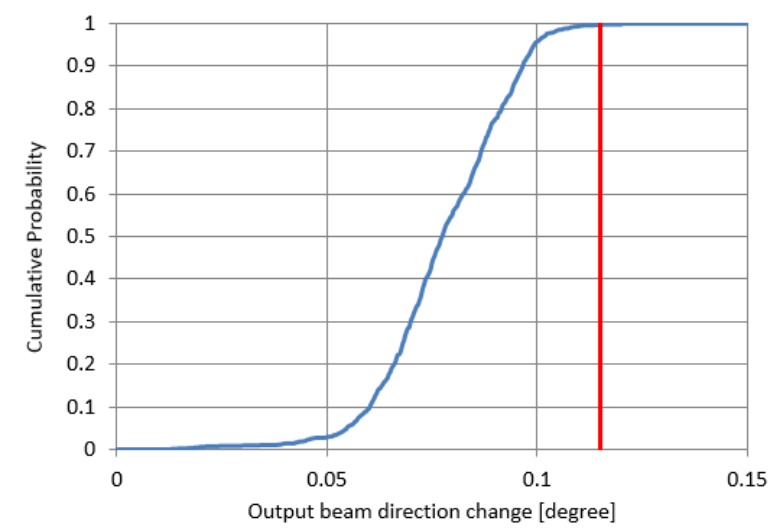

Figure 7. Tolerance analysis result for the flat-mirror design. Red line shows the requirement ( 0.115 degree).

The slicer modules are installed in the spectrograph so that the virtual pseudo-slits locate at the collimator object-side focal surface. In the Slicer-WFOS, the collimator object-side focal surface is matched to the telescope focal surface. But when the slicer modules are used, the image slicers are $\sim 100 \mathrm{~mm}$ away form the telescope focal surface in the default configuration. Hence, the telescope secondary mirror is slightly shifted to match the telescope focal surface to the slicers. It was confirmed that this slight shift of the telescope secondary does not significantly degrade the image quality.

In terms of the image quality, the flat-mirror does not cause any image quality degradation because it consists only of flat mirrors.

\subsection{Design comparison}

Comparison of above three designs are summarized in Table 2. All designs show sufficient image quality, and the flatmirror shows completely no image quality degradation. Two-mirror design exhibits large module tilt sensitivity in the output direction, which is not acceptable for our purpose. Four-mirror design has the much smaller sensitivity, but, if the fabrication and assembly errors are considered, the achievement probability for the requirement is only $\sim 50 \%$. Flatmirror design has negligible module tilt sensitivity in the output beam direction and is expected to meet the specification even if the fabrication and assembly errors are taken into consideration. In addition, its manufacturability is best in these three designs. Although the telescope secondary mirror must be slightly shifted to match the focal plane to the slicer surface, it was confirmed that it does not degrade the image quality in the TMT case. Considering the above excellent performances, the flat-mirror design was selected for the slicer module.

Table 2. Design comparison.

\begin{tabular}{|l|l|l|l|}
\hline Items & Two-mirror & Four-mirror & Flat-mirror \\
\hline \hline Image quality & Slight degradation & Slight degradation & No degradation \\
\hline Output beam direction change & Largest & Larger & Smallest \\
\hline Telescope secondary mirror shift & Not required & Not required & Required \\
\hline Manufacturability & Worse & Worst & Best \\
\hline
\end{tabular}

\section{FIELD CURVATURE OF TMT}

TMT has a steep field curvature with the radius of $\sim 3 \mathrm{~m}$, and the Slicer -WFOS collimator is designed to match this field curvature. Considering the total length of the three pseudo slits $(\sim 42 \mathrm{~mm})$, if the three pseudo-slits are located perpendicularly to the telescope optical axis, the pseudo-slit edges of the two side slices show significant defocus for the collimator in the outer WFOS field (Left panel in Figure 8). To reduce the defocus, the slicer is tilted to align with the same slope of the telescope focal surface. As a result, the virtual pseudo-slits are also parallel to the local slope of the collimator object-side focal surface (Right panel in Figure 8). 

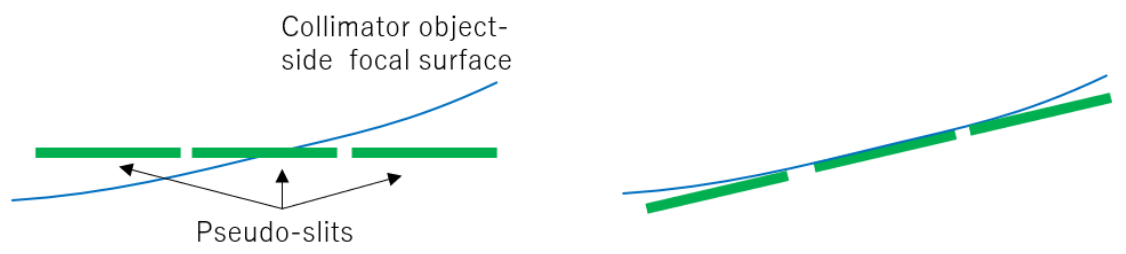

Figure 8. Pseudo-slit location. Three green lines exhibit pseudo-slits and the blue curved line shows the telescope focal surface at the outer region in the Slicer-WFOS field. In the left panel, pseudo-slits are located perpendicularly to the telescope optical axis. In the right panel, they are aligned with the local slope of the telescope focal plane.

This concept requires different kinds of designs for the different field positions. We found that at least 9 kinds of designs are required to cover the entire Slicer-WFOS field. Figure 9 shows the cover regions for the 9 kinds and their tilt angles of the slicer. Figure 10 shows the one example having the 9-degree tilt angle. The central pseudo-slit is offset along the telescope optical axis with respect to the two side pseudo-slits. This is for adapting the field curvature along the Y-axis in Figure 9.

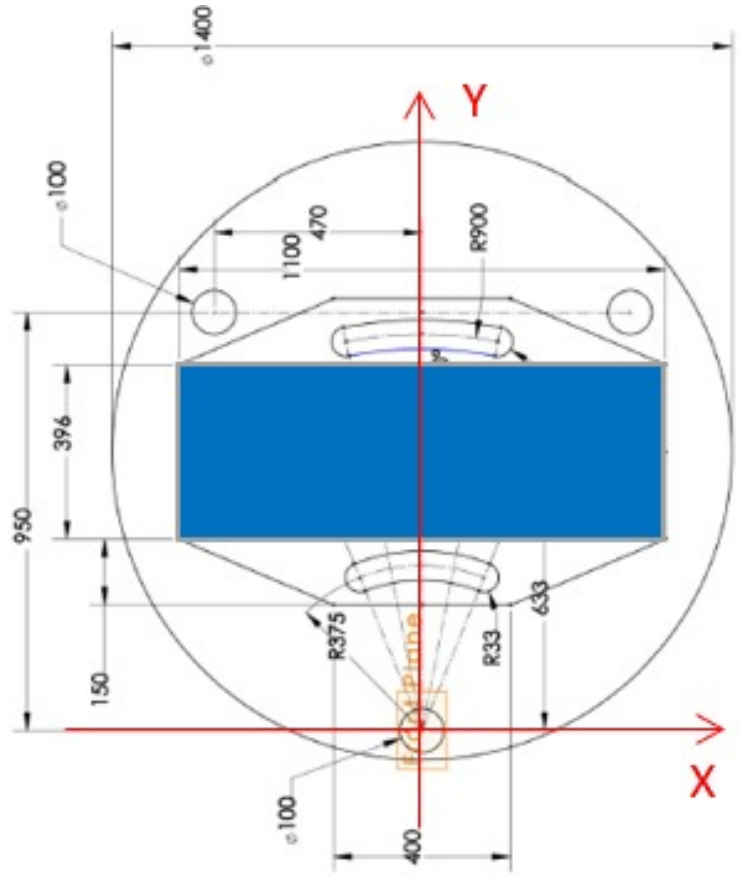

\begin{tabular}{|c|c|c|c|}
\hline $\begin{array}{c}\text { Tilt angle } \\
(\text { degree })\end{array}$ & $\begin{array}{c}\text { Covering } X \text { region } \\
(\mathrm{mm})\end{array}$ & $\begin{array}{c}\text { X length } \\
(\mathrm{mm})\end{array}$ & $\begin{array}{c}\text { Required } \\
\text { module } \\
\text { number }\end{array}$ \\
\hline 9 & $-410--550$ & 140 & 4 \\
\hline 6.9 & $-300--410$ & 110 & 3 \\
\hline 4.8 & $-180--300$ & 120 & 3 \\
\hline 2.4 & $-63--180$ & 117 & 3 \\
\hline 0 & \pm 63 & 126 & 3 \\
\hline-2.4 & $63-180$ & 117 & 3 \\
\hline-4.8 & $180-300$ & 120 & 3 \\
\hline-6.9 & $300-410$ & 110 & 3 \\
\hline-9 & $410-550$ & 140 & 4 \\
\hline & & Total & 29 \\
\hline
\end{tabular}

Figure 9. Left panel shows the Slicer-WFOS field position (blue rectangle) with respect to the telescope optical axis position (XY coordinate origin). The dispersion direction is the $\mathrm{Y}$ direction. Right table summarizes covering $\mathrm{X}$ region for each kind of designs and their required number of modules. 


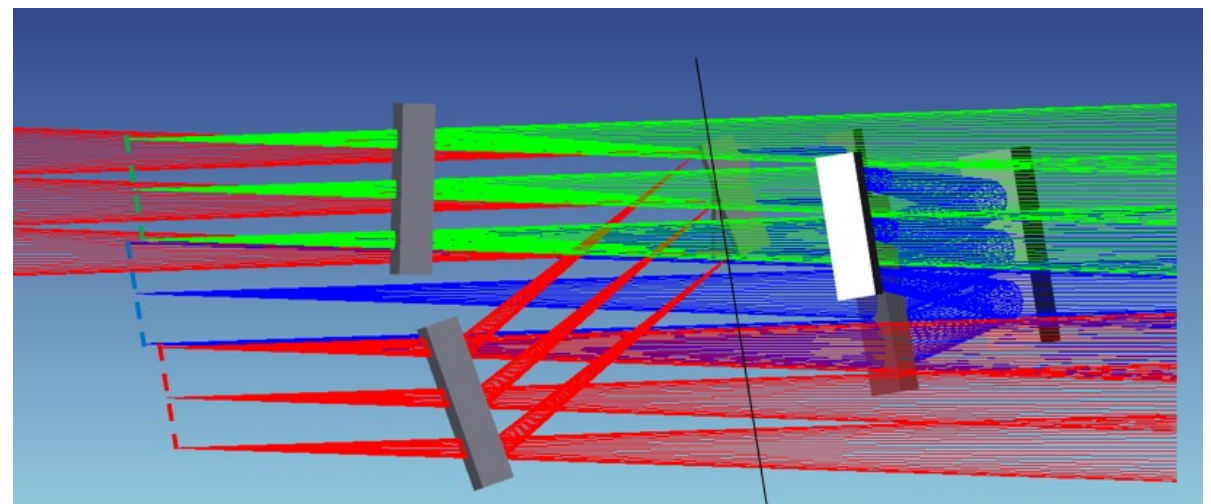

Figure 10 Tilted design example. Black line shows the slicer surface. Colored dashed lines show the virtual pseudo-slits.

\section{ACKNOWLEDGEMENTS}

The Authors would like to thank Bernard Delabre for providing the original idea of Flat-mirror design.

The TMT Project gratefully acknowledges the support of the TMT collaborating institutions. They are the California Institute of Technology, the University of California, the National Astronomical Observatory of Japan, the National Astronomical Observatories of China and their consortium partners, the Department of Science and Technology of India and their supported institutes, and the National Research Council of Canada. This work was supported as well by the Gordon and Betty Moore Foundation, the Canada Foundation for Innovation, the Ontario Ministry of Research and Innovation, the Natural Sciences and Engineering Research Council of Canada, the British Columbia Knowledge Development Fund, the Association of Canadian Universities for Research in Astronomy (ACURA), the Association of Universities for Research in Astronomy (AURA), the U.S. National Science Foundation, the National Institutes of Natural Sciences of Japan, and the Department of Atomic Energy of India.

\section{REFERENCES}

[1] Bernstein, R. A. and Bigelow, B. C., "An Optical Design for a Wide-Field, Optical Spectrograph for TMT," Proc. SPIE, 7014, 70141G (2008)

[2] Bigelow, B. C. and Bernstein, R. A., "Progress on the Conceptual Design for the MOBIE Imaging Spectrograph for the Thirty Meter Telescope," Proc. SPIE, 7735, 773527 (2010)

[3] Bigelow, B. C., Radovan, M. V., Bernstein, R. A., Onaka, P. M. Yamada, H., Isani, S., Miyazaki, S. and Ozaki, S., "Conceptual Design of the MOBIE Imaging Spectrograph for TMT," Proc. SPIE, 9147, 914728 (2014)

[4] Bundy, K. et al., "WFOS instrument trade study: slicer vs. fiber instrument concept designs and results," Proc. SPIE, 10702, in press (2018) 